\title{
THE STARBURST REGION 30 DORADUS
}

\author{
NOLAN R. WALBORN ${ }^{1}$ \\ Space Telescope Science Institute ${ }^{2}$ \\ 3700 San Martin Drive \\ Baltimore, Maryland 21218, USA
}

\begin{abstract}
The 30 Doradus region of the LMC is a Rosetta Stone for the interpretation of similar, more distant regions, since it is accessible to spatially resolved investigation of its components with modern instrumentation. Following an introductory review of the various scales and populations relevant to the region, several current programs which promise improved understanding will be reviewed. They include extensive new optical spectroscopy and photometry of the central cluster, which will provide substantial new information about the stellar content and initial mass function (IMF); infrared surveys which will clarify questions about continuing star formation and the lower main sequence; and high-resolution nebular spectroscopy and X-ray observations which will reveal the high-energy phenomena in the surrounding interstellar medium.
\end{abstract}

\section{Introduction}

An understanding of the structure and content of 30 Doradus is an essential prerequisite to the interpretation of more distant starburst regions. Paradoxically, we already know far more about 30 Doradus than can be reviewed in the present time and space, and yet several of its most fundamental aspects are only now being revealed by investigations in progress. The principal reason is that, although 30 Doradus is much more accessible to detailed study than any other starburst, it still represents a difficult observational problem at the limit of a given instrumentation, so that any advance in resolution, signal-to-noise, and/or wavelength coverage immediately yields significant new information. Therefore, rather than attempting to cover all recent developments, I shall, following a brief survey of the relevant spatial scales and stellar populations, concentrate on three current areas of research which in my judgment will provide substantial new insights into the nature of this spectacular region.

1 Visiting Astronomer, Anglo-Australian Telescope.

2 Operated by the Association of Universities for Research in Astronomy, Inc., under contract with the National Aeronautics and Space Administration. 


\section{Scales and Populations}

In comparing 30 Doradus with more distant objects, it is important to distinguish, in descending order of size, the scales of the 30 Dor region, nebula, ionizing cluster, and central object (R136). The region is usually taken to encompass Henize (1956) N158/N159/N160 to the south, with a scale of about $1 \mathrm{kpc}$, although on many photographs the twice greater region of N135 appears as a coherent entity containing 30 Dor (N157) and the other nebulosities (Hodge and Wright 1967, Elliott et al. 1977). More than half of the LMC WR stars listed by Breysacher (1981) are within N135, as are large numbers of B and M supergiants, minus one which was the SN 1987A progenitor. Of course, some of this noncoeval population will be projected onto, or even contained within, the 30 Dor Nebula, which has a diameter of about $200 \mathrm{pc}$ (Walborn 1984). The 30 Dor ionizing cluster, defined by the distribution of the very early $O$ stars (Melnick 1985, Walborn 1986), extends about $3^{\prime}$ or $40 \mathrm{pc}$, which also corresponds to the extent of the brightest Tarantula filaments. The luminous central object R136 (Feast, Thackeray, and Wesselink 1960) is now well established as the dense core of the ionizing cluster, containing 27 resolved components (Walborn 1973; Walker and O'Donoghue 1984; Moffat, Seggewiss, and Shara 1985; Weigelt and Baier 1985; Neri and Grewing 1988). Figure 1 is a shallow cut from a remarkable CCD image with 0 !' 6 resolution obtained at the ESO $2.2 \mathrm{~m}$ (kindly made available by Dr. G. Meylan), which shows by inspection the outer structure of R136a previously seen only in the maximum-entropy and speckle analyses: supermassive stars have five points! One awaits with anticipation even better images from the ESO NTT and the HST. Such dense cores may be a characteristic of the formation of massive starbursts, as evidenced by the related galactic objects NGC 3603 (Walborn 1973, Moffat et al. 1985) and W49 (Welch et al. 1987). These various scales are summarized in Table 1 , both in linear measure, and in angular for the LMC and greater distances. Perhaps the most noteworthy entry implies that the intricacies of the 30 Dor ionizing cluster, to be further discussed below, will subtend less than $1^{\prime \prime}$ in the Virgo Cluster, not to mention even more distant systems.

TABLE 1. Characteristic Dimensions

\begin{tabular}{lcccc}
\hline & Linear & \multicolumn{3}{c}{ Angular } \\
\cline { 3 - 5 } & & $52.5 \mathrm{kpc}$ & $1 \mathrm{Mpc}$ & $10 \mathrm{Mpc}$ \\
\hline LMC & $5 \mathrm{kpc}$ & $5^{\circ}$ & $15^{\prime}$ & $1.5^{\prime}$ \\
30 Doradus Region & $1 \mathrm{kpc}$ & $1^{\circ}$ & $3^{\prime}$ & $20^{\prime \prime}$ \\
30 Doradus Nebula & $200 \mathrm{pc}$ & $15^{\prime}$ & $50^{\prime \prime}$ & $5^{\prime \prime}$ \\
30 Doradus Cluster & $40 \mathrm{pc}$ & $3^{\prime}$ & $10^{\prime \prime}$ & $1^{\prime \prime}$ \\
R136 & $2.5 \mathrm{pc}$ & $10^{\prime \prime}$ & $0.5^{\prime \prime}$ & $0.05^{\prime \prime}$ \\
R136a & $0.25 \mathrm{pc}$ & $1^{\prime \prime}$ & $0.05^{\prime \prime}$ & $0.005^{\prime \prime}$ \\
\hline
\end{tabular}

Table 2 summarizes the outstanding properties of four distinct evolutionary stages of massive young regions. The $\mathbf{3 0}$ Dor ionizing cluster definitely corresponds to the Carina phase, but all four populations are present in the field of the 30 Dor Nebula, and the question is, which if any of the other three are generically related to the ionizing cluster? As discussed in Section 4 below, there is now very strong evidence for sequential formation of a physically associated Orion phase; on the other hand, a physically associated $h$ and $\chi$ Per phase can be excluded, as was already discussed by Walborn (1984). The relatively 
Figure 1. Ten-sec B CCD exposure of the 30 Dor cluster from the ESO $2.2 \mathrm{~m}$ by Dr. G. Meylan. North is at top and east to the left; the extent in declination is $2^{\prime}$ and the resolution is $0 ! ' 6$. Note the structure of $\mathrm{R} 136 \mathrm{a}$, the bright composite object at the center.

numerous late-O/early-B supergiants (Sco OB1 phase) present a more difficult problem, however; as shown by Walborn (1986) and discussed by Moffat et al. (1987), they are less concentrated toward R136 than the earlier types, but further work is required for a definitive answer.

\section{Optical Spectroscopy and Photometry: The Current Generation}

Spectroscopic knowledge of the 30 Dor ionizing cluster as of that time (to about 14th mag) was summarized by Melnick (1985) and Walborn (1986), who showed that it constitutes 
TABLE 2. Morphology of Massive Young Regions

\begin{tabular}{llcccccc}
\hline & \multicolumn{2}{c}{$\begin{array}{c}\text { Visually } \\
\text { Region }\end{array}$} & Brightest Stars & \multicolumn{2}{c}{ MS Turnoff } & & H II, \\
\cline { 2 - 4 } & Spectrum & $\sim$ Mass & $\sim$ Age & Dust & Red Sg \\
\hline Orion Nebula & ZAMS O, (IR) & $($ PMS $)$ & - & $<10^{6}$ yrs & Yes & No \\
Carina Nebula & O3, WN & O3 & $100 M_{\odot}$ & $3 \times 10^{6}$ & Yes & No \\
Scorpius OB1 & OB Sg & O6-7 & 60 & $4-5 \times 10^{6}$ & No & No \\
$h+\chi$ Persei & A Sg & O9-B0 & 20 & $8-9 \times 10^{6}$ & No & Yes \\
\hline
\end{tabular}

the most spectacular concentration of hot, massive stars for which detailed information is available. The cluster includes five examples of the remarkable, rare $\mathrm{O3}$ If/WN-A supergiant category, with apparent magnitudes similar to those of the brightest unresolved speckle components within $\mathrm{R} 136 \mathrm{a}$, which are probably similar objects and strong candidates for the most massive stars known. In collaboration with $\mathrm{C}$. Blades, I have undertaken to extend this information to about magnitude 15.5, by means of the AAT multiple-object, fiber-optics system, in order to investigate whether later spectral types or somewhat more reddened very early types predominate at that level. The results of a first-pass classification of these data are summarized in Table 3, which shows that, along with the significant number of later types found, the number of known very early types has been doubled by this study. Most or all of the evolved B- and A-type objects are probably nonmembers of the ionizing cluster. In addition to the 111 OBA types, $11 \mathrm{WRs}$ exclusive of R136 (Moffat et al. 1987) and 13 late-type spectra (a mixture of LMC supergiants and foreground dwarfs/giants) are known in the field. However, Figure 1 shows many crowded objects of similar magnitudes which have not yet been observed spectroscopically, and R136 contains at least 27 components brighter than 16th mag which are unknown spectroscopically, except for two additional WRs.

In addition to the statistical information, as is usually the case in studies of this kind, the new observations have revealed a number of individual objects of special astrophysical interest. They include some probable very early evolutionary stages (Walborn and Blades 1987; Section 4 below), very early-O giants, the first $O(n)(f)$ p spectrum in the LMC, a new heavily-reddened Of supergiant (Walborn 1990), B supergiants with CNO anomalies, and a variable B-type spectrum with an apparent He II $\lambda 4686$ emission line (Section 5 below).

Currently, several CCD programs are underway which will soon provide accurate photometry for most stars in the field for the first time. Perhaps the most extensive is by J. Parker, C. Garmany, and P. Massey, but other studies are also in progress (separate private communications from A. Campbell, G. Meylan, and A. Moffat). In addition to permitting evaluation of the reddening and absolute magnitudes of the spectroscopically classified stars, their work will contribute vital information about the IMF and the lower main sequence (if it is observable), although for the former purpose combination with the spectral types is essential (Massey, Parker, and Garmany 1989). A discussion of 30 Doradus in relation to the LMC blue globular clusters has been presented by Kennicutt and Chu (1988), and to the integrated LMC IMF by Blaha and Humphreys (1989).

The hydrogen-ionizing luminosity of the spectroscopically classified $O$ and WR stars in the 30 Dor cluster (exclusive of R136) has been computed following the procedures of Walborn (1984), the result being $2.8 \times 10^{51} \mathrm{sec}^{-1}$. Fortuitously, this result is essentially 
TABLE 3. The Known OB Stellar Content of 30 Doradus

\begin{tabular}{lccc}
\hline $\begin{array}{l}\text { Spectral } \\
\text { Types }\end{array}$ & $\begin{array}{c}\text { Melnick, } \\
\text { Walborn }\end{array}$ & $\begin{array}{c}\text { Walborn \& } \\
\text { Blades }\end{array}$ & TOTAL \\
\hline O3-6 V & 14 & 17 & 31 \\
O3-6 III & 3 & 3 & 6 \\
O3 If* & 5 & 0 & 5 \\
O6-9 V & 4 & 11 & 15 \\
O6-9 III & 5 & 10 & 15 \\
O6-8 Iaf & 1 & 1 & 2 \\
O9 I & 2 & 1 & 3 \\
& & & \\
B0-1 V & 0 & 1 & 1 \\
B0-1 III & 0 & 7 & 14 \\
B0-1 I & 11 & 3 & 2 \\
B1-3 III & 0 & 2 & 3 \\
B1-3 I & 1 & 2 & 7 \\
B5-A I & 4 & 3 & 111 \\
TOTAL & & & \\
\hline
\end{tabular}

identical to that obtained in the earlier analysis based primarily on approximate photometry alone, although that estimate involved 136 stars while the present one contains 88 ; the reason is that some of the former turned out to be of type $B$, while others are of earlier $O$ types than had been expected. The earlier estimate of $2 \times 10^{51} \mathrm{sec}^{-1}$ for R136a cannot yet be improved upon. For comparison, the H II luminosity within a $140 \mathrm{pc}$ diameter is $8.7 \times 10^{51} \mathrm{sec}^{-1}$ (Mills, Turtle, and Watkinson 1978). Several sources for the "missing" ionization can be readily suggested: early-type members of the central cluster not yet classified spectroscopically (see above); higher than assumed ionizing luminosities for the WR stars; early-type stars in the periphery of the Nebula, not included in the central cluster count (e.g., R130, R144, R146, R147, Breysacher 73; Testor and Schild 1990 have just announced the discovery of a new 03 If/WN-A near the latter, emphasizing anew that the census of even the most spectacular stellar component in this region remains incomplete); and shocks or soft X-rays (Section 5 below). Discussions of the global energetics of 30 Doradus have recently been published based upon the UV (Smith, Cornett, and Hill 1987), H $\alpha$ (Kennicutt and Hodge 1986), IRAS (Schwering 1989), CO (Cohen et al. 1988), and radio continuum (Klein et al. 1989).

\section{Infrared: New Beginnings}

The dominant optical components of the 30 Doradus Nebula and ionizing cluster are evolved: the most massive stars have reached the WR stage, implying ages of two to three million years (Maeder 1983), and the nebulosity appears to be expanding away from the cluster (e.g., Walborn 1980). Nevertheless, an $\mathrm{H}_{2} \mathrm{O}$ maser source has been discovered in the northeast quadrant of the Nebula (Whiteoak et al. 1983, Whiteoak and Gardner 1986). Walborn and Blades (1987) reported two early O-type objects in dense nebular knots, one 
within the maser positional error circle and the other nearby within the brightest Tarantula filament, and suggested that they may represent a significantly earlier evolutionary stage. There are several other prominent nebular knots in this sector which may contain fainter embedded stellar objects. Subsequently, a third essentially identical stellar/nebular knot has been found within the second-brightest Tarantula filament immediately west of R136; although not marked, it is easily seen in Fig. 1 of Walborn and Blades (1987).

In a remarkable IR imaging survey of the 30 Dor Nebula, Hyland et al. (1990; see also Jones et al. 1986) have found definitive evidence for current massive star formation in the form of four luminous protostars, three of which are located in the northeast quadrant near the maser and optical knots, while the fourth is just a few arcsec from the new stellar/nebular knot west of R136. The morphology strongly suggests that the energetic activity of the central ionizing cluster has initiated a new stellar generation in these surrounding areas, and this region now becomes one of the strongest cases for sequential star formation known. The protostars have extremely red J-K colors and featureless, red $2 \mu$ spectra, and they are significantly above the survey magnitude limit, suggesting a possible deficiency of lower masses. These results presage a substantial advance in our understanding of 30 Doradus and massive star formation; one awaits with anticipation further images from the new IR arrays.

\section{High Energy: Last Things}

The morphology of massive young regions implies that rich $O B$ clusters disperse their remanent gas and dust on the evolutionary timescale of the most massive stars (Table 2). The presumed agents of this dispersal are the high-energy stellar radiation, winds, and supernova events. Direct evidence of this activity is provided by high-velocity nebular kinematics and soft $\mathrm{X}$-ray emission.

The moderate-velocity, large-scale expansion(s) of 30 Doradus (of order $10 \mathrm{~km} / \mathrm{sec}$ ) have been discussed by, e.g., Cox and Deharveng (1983), Meaburn (1984), and Clayton (1987). However, there is also evidence for numerous smaller expanding shells with much higher velocities (of order $100 \mathrm{~km} / \mathrm{sec}$ ) throughout the Nebula (Meaburn 1984; Chu and Kennicutt 1988; Chu, private communication); one striking example has been discussed in detail by Meaburn (1988). The latter object is centered on the small $h$ and $\chi$ Pertype cluster, containing $\mathrm{B}, \mathrm{A}$, and $\mathrm{M}$ supergiants, $3^{\prime}$ northwest of $\mathrm{R} 136$. In the AAT stellar spectroscopic study (Section 3 above), a variable B-type spectrum with probable He II $\lambda 4686$ emission has been found in this cluster; it may be considered a candidate for the (binary) stellar remnant of a SN which produced the high-velocity shell.

The Einstein Observatory detected 30 Doradus as an extended, soft X-ray source with remarkable structural correspondences to the optical nebular morphology, as shown in Figure 2 (see also Harnden and Seward 1988); detailed analyses of these data have just recently appeared, yielding a luminosity of order $10^{37} \mathrm{ergs} / \mathrm{sec}$ and a total thermal energy of $10^{52}$ ergs (Chu and MacLow 1990, Wang and Helfand 1990). The X-ray emission and the high-velocity gas motions are undoubtedly related, since they have similar energy contents, as is the case in the Carina Nebula (Seward and Chlebowski 1982, Walborn and Hesser 1982, Walborn 1982). Following the procedures in the latter reference, the kinetic energy from the stellar winds of the spectroscopically classified $O$ and WR stars in 30 Dor has been estimated as $10^{52}$ ergs; galactic wind parameters were used since extensive determinations 


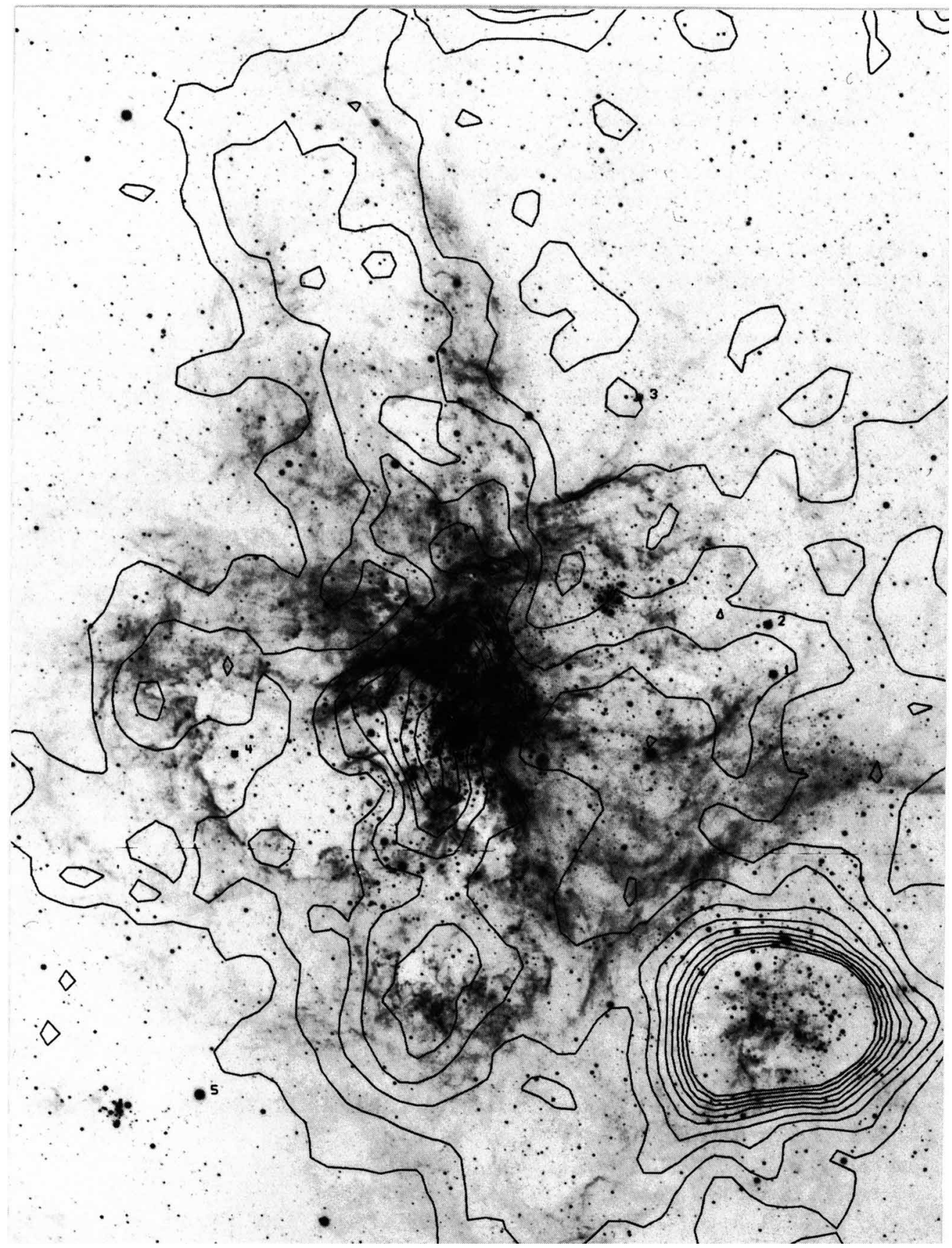

Figure 2. Einstein Observatory IPC X-ray contours provided by Dr. F. Seward, superposed on the [S II] optical image of 30 Dor described by Walborn (1984). 
as a function of spectral type for LMC stars are not yet available, but on the other hand the R136 components and other unclassified members of the ionizing cluster are not included. As is the case in Carina, the stellar winds are probably capable in principle of generating the high-energy phenomena observed in the Nebula, but it is likely that embedded SNR contribute as well in 30 Doradus.

\section{References}

Blaha, C. and Humphreys, R. M. 1989, A. J., 98, 1598.

Breysacher, J. 1981, Astr. Ap. Suppl., 43, 203.

Chu, Y.-H. and Kennicutt, R. C., Jr. 1988, A. J., 86, 1874.

Chu, Y.-H. and MacLow, M.-M. 1990, Ap. J., in press.

Clayton, C. A. 1987, Astr. Ap., 173, 137.

Cohen, R. S., Dame, T. M., Garay, G., Montani, J., Rubio, M., and Thaddeus, P. 1988, Ap. J. (Letters), 331, L95.

Cox, P. and Deharveng, L. 1983, Astr. Ap., 117, 265.

Elliott, K. H., Goudis, C., Meaburn, J., and Tebbutt, N. J. 1977, Astr. Ap., 55, 187.

Feast, M. W., Thackeray, A. D., and Wesselink, A. J. 1960, M.N.R.A.S., 121, 337.

Harnden, F. R., Jr. and Seward, F. D. 1988, in Fourth George Mason Astrophysics Workshop, Supernova 1987A in the Large Magellanic Cloud, ed. M. Kafatos and A. G. Michalitsianos (Cambridge), p. 408.

Henize, K. G. 1956, Ap. J. Suppl., 2, 315.

Hodge, P. W. and Wright, F. W. 1967, The Large Magellanic Cloud (Smithsonian Publ. 4699).

Hyland, A. R., Straw, S., Jones, T. J., and Gatley, I. 1990, in preparation.

Jones, T. J., Hyland, A. R., Straw, S., Harvey, P. M., Wilking, B. A., Joy, M., Gatley, I., and Thomas, J. A. 1986, M.N.R.A.S., $219,603$.

Kennicutt, R. C., Jr. and Chu, Y.-H. 1988, A. J., 95, 720.

Kennicutt, R. C., Jr. and Hodge, P. W. 1986, Ap. J., 306, 130.

Klein, U., Wielebinski, R., Haynes, R. F., and Malin, D. F. 1989, Astr. Ap., 211, 280.

Maeder, A. 1983, Astr. Ap., 120, 113.

Massey, P., Parker, J. W., and Garmany, C. D., 1989, A. J., 88, 1305.

Meaburn, J. 1984, M.N.R.A.S., 211, 521. . 1988, M.N.R.A.S., 235, 375.

Melnick, J. 1985, Astr. Ap., 153, 235.

Mills, B. Y., Turtle, A. J., and Watkinson, A. 1978, M.N.R.A.S., 185, 263.

Moffat, A. F. J., Niemela, V. S., Phillips, M. M., Chu, Y.-H., and Seggewiss, W. 1987, Ap. J., 312, 612 .

Moffat, A. F. J., Seggewiss, W., and Shara, M. M. 1985, Ap. J., 295, 109.

Neri, R. and Grewing, M. 1988, Astr. Ap., 196, 338.

Schwering, P. B. W. 1989, Astr. Ap. Suppl., 79, 105.

Seward, F. D. and Chlebowski, T. 1982, Ap. J., 256, 530.

Smith, A. M., Cornett, R. H., and Hill, R. S. 1987, Ap. J., 320, 609; err. ibid. 355, 746 (1990). 
Testor, G. and Schild, H. 1990, Astr. Ap., in press.

Walborn, N. R. 1973, Ap. J. (Letters), 182, L21. . 1980, Ap. J. (Letters), 235, L101. . 1982, Ap. J. Suppl., 48, 145. 1984, in IAU Symp. 108, Structure and Evolution of the Magellanic Clouds, ed. S. van den Bergh and K. S. de Boer (Reidel), p. 243. 1986, in IAU Symp. 116, Luminous Stars and Associations in Galaxies, ed. C. W. H. de Loore, A. J. Willis, and P. Laskarides (Reidel), p. 185. . 1990, in ASP Conf. Ser. Vol. 7, Properties of Hot Luminous Stars, ed. C. D. Garmany (BookCrafters), p. 23.

Walborn, N. R. and Blades, J. C. 1987, Ap. J. (Letters), 323, L65.

Walborn, N. R. and Hesser, J. E. 1982, Ap. J., 252, 156.

Walker, A. R. and O'Donoghue, D. E. 1984, Astr. Express, 1, 45.

Wang, Q. and Helfand, D. J. 1990, preprint.

Weigelt, G. and Baier, G. 1985, Astr. Ap., 150, L18.

Welch, Wm. J., Dreher, J. W., Jackson, J. M., Terebey, S., and Vogel, S. N. 1987, Science, 238, 1550.

Whiteoak, J. B. and Gardner, F. F. 1986, M.N.R.A.S., $222,513$.

Whiteoak, J. B., Wellington, K. J., Jauncey, D. L., Gardner, F. F., Forster, J. R., Caswell, J. L., and Batchelor, R. A. 1983, M.N.R.A.S., 205, 275. 